\title{
Sensor-based mobile pigeonhole alert system
}

\begin{abstract}
Information system is an essential aspect of any organization which help with the dissemination and retrieval of information among staff members. Most organizations experience ineffective information management due to the use of ageing information processing methods and poor staff management. There has been an improvement with the adoption of conventional pigeonholes as message boxes allocated to each member to track staff incoming and outgoing mails. In this approach, organizations are still confronted with untimely acknowledgement of incoming mails, delivery and feedback. This paper proposes a mobile pigeonhole alert system with the use of a sensor device to send an alert to the intended pigeonhole users notifying them of the arrival of a message. Hardware and Software implementations were carried out using JAVA programming language to program a sensor, hardcoded on a micro-controller. To assess the effectiveness of the system, an online survey was carried out to elicit information from two hundred (200) pigeonhole users (lecturers) in a tertiary institution about their perceptions of the system usability and performance. The data collected were analyzed using SPSS software for correlation and regression analysis between the usability and system performance. Experimental results showed that system performed as expected based on users' satisfaction, realization of system objectives and the significant values obtained from statistical analysis. Comparative analysis of the system also showed an improvement on other existing works.
\end{abstract}

Keywords: pigeonhole, alert system, information exchange, messages, communication, sensor device
Volume 6 Issue I - 2020

\section{Taiwo Gabriel OMOMULE, Olusola Olajide AJAYI, Sekinat Bolanle ADEKILE \\ Department of Computer Science, Adekunle Ajasin University, Nigeria}

Correspondence: Olusola Olajide AJAYI, Department of Computer Science, Adekunle Ajasin University, P.M.B.00I,Akungba-Akoko, Ondo State, Nigeria, Email olusola.ajayi@aaua.edu.ng

Received: January 23, 2020 | Published: January 3I, 2020

\section{Introduction}

The purpose of an information system is processing, memorizing and transmitting appropriate information in place, and the best information system is one that performs this function effectively within little computational cost and time. All systems including computer systems consist of accepting raw data as inputs, using stored programs for processing the data and producing outputs as timely information. The process of transforming inputs into outputs is known as information management. ${ }^{1}$ One approach which organizations can utilize computing capability is through the development of Management Information Systems (MIS). MIS is a system using formalized procedures to provide management at all levels in all functions with appropriate information based on data from both internal and external sources, to enable them to make timely and effective decisions for planning, directing and controlling the activities for which they are responsible. It will be noted from the above definition that the emphasis is on the uses to which the information is put. Planning, directing and controlling are the essential ingredients for management. ${ }^{2}$ In essence, the processing of data into information and communicating the resulting information to the user is the key function of MIS. It should therefore be noted that MIS exist in organizations in order to help them achieve objectives, to plan and control their processes and operations, to help deal with uncertainty, and to help in adapting to change or, indeed, initiating change. Management Information System (MIS) is one of the most important tools in any organization, which aims to provide reliable, complete, accessible, and understandable information in a timely manner to the users of the system. ${ }^{3}$ MIS is a flow of procedures for data processing based on the computer, and integrated with other procedures in order to provide information in a timely and effective manner to support decision making and other management functions. ${ }^{4}$ The authors $\mathrm{in}^{5}$ opined that in order for organizations to advance into the future, they must adopt the technology utilization approach, which is a mandatory requirement for such organizations which seek excellence in performance. Moreover, the importance of MIS comes from the benefits that are generated by that system such as providing useful information in a timely manner, improved labor productivity, cost savings, providing the information without any delays and mistakes, and improved the management of work. ${ }^{3}$ One of the effective ways of managing information dissemination and retrieval in an organization is the introduction of Pigeonhole concept where information in the form of mails are delivered for the perusal and usage of different staff in the organization.

Pigeonhole is an internal information exchange system, used for communication in an organization. It is a creative, informal and traditional way of dropping and picking messages in a set of small open-fronted compartments usually used in a workplace or other organizations where letters or messages may be left for individuals. The chain of exchange is a kind of 'give-and-return'. It is a bidirectional 'pick-and-respond' information transmission mode. ${ }^{6}$ It can exist in wooden box or metal shelf or take any other form with several rectangular holes in it which is usually been used in offices or large organizations. Pigeonhole works similar to letterbox where letter or memo for specific person will be placed in their letterbox. The author's in ${ }^{6}$ stated that each staff in most big organization has his/ her own pigeonhole to receive any important letter or memo related to the official duty. Each staff has been allocated a pigeonhole for any letters or memos from within or outside the department, unit or faculty. Unfortunately, the current conventional pigeonhole system is unable to inform the staff on any urgent letter and this leads to 
significant delay in responding to the letter. The main weakness of the current system is that staff needs to check their respective pigeonhole everyday but due to the routine commitment or unforeseen circumstances, the pigeonhole cannot be possibly checked every day. The system proposed in $^{6}$ intends to computerize the traditional way of dropping and picking messages in a set of small open fronted compartments usually used in a workplace or other organization with a two-way communication between the office clerk and the staff via a mobile application. The clerk sends messages via his/her mobile app to the staff notifying them of the availability of memos, letters and other documents in their pigeonhole, the intended staff sends feedback concurrently. Several problems have been identified in this method as untimely delivery and responses to messages, poor feedback, time and effort are wasted in checking up pigeonholes on daily-basis and sometimes clerk's unavailability. Therefore, this paper proposes a pigeonhole alert system using sensor device as an improvement to the stated problems in the proposed system by ${ }^{6}$ to detect the presence of a mail in the pigeonhole with the use of a sensor device and a short messaging system to alert the staff on their mobile phone of the need to pick up their mails.

\section{Related works}

Several researchers have proposed different techniques and technology to enable timely delivery and access of information across various platforms with notable contributions and limitations. The authors in ${ }^{7}$ proposes an SMS service system for student collaboration on campus. The research focuses on quick message communication and delivery among students on campus. Simple wire wireless message protocol and ActiveX SMS software development kit for visual basic serve as the tools used to develop the system. The system achieve messaging objective but lacks mobility access via phone and deployment cost was high. An SMS technology that supports classroom interaction between students and lecturers is proposed in ${ }^{8}$ The aim was to bridge the communication between lecturers and students in a classroom environment. Students send SMS via their mobile phone which are viewed, replied and addressed by the lecturers through a developed software connect with a modem. A class website developed is used as the interactive platform between the students and the lecturer after which the SMS has been published on the website. The implementation of the system was characterized by Java to develop a GUI that model the users' real mobile phone. However, cases of failure in remote connection between the students and lecturers were recorded.

$\mathrm{In}^{9}$ a vision-based on-line traffic information system is presented. The system was to monitor and detect levels of traffic congestion on certain roads in Dhaka City and to make this information available to the travelers. The system was implemented using a multiple Web Cams which is installed on designated roads. The system will capture digital images of the traffic, analyze these images and reach a clear decision about number of cars. Users will then be able to reach this data by using the short messaging service in their mobile phones. Basically, the system is divided into three independents, yet interacting modules: the image capturing module which will automate the capture of images, the digital image processing module which will process the images and the short message service (SMS) server module which will receive SMSs from a user and reply back to him by an SMS. An m-banking system using the m-commerce technology for the banking sector is presented in $^{10}$ the aim was to provide a mobile banking platform that provides several essential banking services to customers by only sending SMS to bank server from any remote location. This proposed system was implemented by dividing it into five modules: Interfacing Module, SMS Technology Adoption Module, SMS Banking Registration Module, Service Generation Module and Data Failover Module. Four major services such as balance enquiry, balance transfer between authenticated customers, deposit payment and bill payment were provided without physically going to bank thereby saving customers' time.

The authors in ${ }^{11}$ proposes a Short Message Service using SMS Gateway. The system suggested a multi-level local authentication to the SMS gateway service. A web interface and an encryption method. The application and mobile carriers are connected via TCP/ IP. SMPP was used as the SMS protocol that is secure and sustain greater message volumes $10,000 / \mathrm{min}$. The software was designed in Visual Basic 2005.The database connection was written using Query based on SQL. SQL was used to store the record and also to retrieve the record. However, there were inadequacies in messaging functionalities and latest encryption algorithm was not adopted. An SMS application system along with the corresponding server is developed by the authors $i^{5}$ the aim was to provide a system that can avoid the reliance of Content Delivery SMS application of student examination result to SMS Gateway Provider and the commercial SMS application developer which can be managed totally by the school staff. The Rational Unified Process (RUP) methodology is implemented in this research where the researcher could iteratively do the system development during each phase. The system promoted SMS technology in school However, cases of network traffic on the server affects system performance at peak times.

A mobile interface for Community Health Information Tracking System (CHITS) is proposed in $^{12}$ the motivation was to suggest an approach for cheap and effective remote connection to the CHITS server enabling the synchronization of data in real time. The proposed system was developed with JAVA2ME. The Data was collected and compressed using the jgz java library with deflate based compression algorithm to minimize the amount of SMS messages to be sent. SMSLib serves as a platform through which a server phone was connected to the server to receive messages through designed protocol that assures availability and reliability. A cost effective way of transferring data remotely with the use of SMS was established but there were limitations in the amount of data that an SMS can carry and manual copying of database file when not using the remote mode. A framework for the design of a Mobile-Based Alert System for Outpatient Adherence in Nigeria is proposed. ${ }^{13}$ The aim was to ensure adherence to long-term therapy in outpatient condition to ensure effective treatment and reduce or curb the prevalence of these diseases. A system for mobile technology that will provide an easy way of complying with drug regimen was developed. The system utilizes Short Messaging Service (SMS) via mobile phones to provide reminders at dosing times. However, the system was limited by the inability to deploy and evaluate the prototype application within its scope so the system was not tested.

The authors $\mathrm{in}^{14}$ proposes a Short Messaging Service as an Alternative for Pushing Information to Build Efficient Information Passing Systems in Academic Institutions. The system was targeted at improving existing levels of communication between teachers and students of an Academic Institution. A total solution architecture was proposed. The architecture consists of a Central Database Server to store and forward requests, a networking interface to send SMS'es 
successfully and a client end application to read and acknowledge the same. The system was implemented using Open Source API's and a middleware one can build a service wherein students don't have to pay for Student Information Services. The System provided a high degree of security and confidentiality and also generate timely information needed in Decision Support System of the institution. However, there are possibilities for network failure and response of the entire architecture therein. In $^{15}$ a smart pigeonhole system by sending notification through short messaging system is presented. The aim was to develop a time-saving system for resident to get a notification about arriving mail through short messaging system on their phone. The system was implemented using several hardware components including infrared sensors, ultrasonic sensors and arduino Yun board. Infrared sensors were used to detect the presence of the mail into the box while the ultrasonic sensor was used to detect the level of fullness of the mailbox. The system achieved SMS notification for pigeonhole mail arrivals but the sensor could only be powered by electricity. Cases of power outage followed by subsequent mail arrival would limit the system functionality.

$\mathrm{In}^{16}$ the design and development of electronic pigeonhole system integrated with GSM network to send a notification of any upcoming loaded is presented. The system was needed to send short message service (SMS) notification to designated users when a new letter is placed in their pigeonhole. The system was developed by integrating a detection circuit that contain voltage regulator, Infrared sensors and microcontrollers to acknowledge the existence of new post items with the GSM modem to transmit SMS to specific user. The system was attached to a metal pigeonhole and tested. The system provided immediate notifications to intended users for further action. However, the implementation was too costly and there is no possibility of performing system test on wooden platform. An SMS tool to exchange information in medical area was proposed. ${ }^{17}$ The work was developed as a Radiological Information System (RIS) where physician can send messages to their patient. RIS system can be configured to send SMS when the examination is scheduled and to send another SMS later to remind the patient of the appointment. The system offered an easy medium for timely information delivery in the research domain. The authors in ${ }^{18}$ developed an integrated e-parcel management system using GSM network. The system notifies user of the upcoming parcel reach at university via SMS. Their work offered robust platform that enhanced quick message delivery and retrieval that is useful for the day-to-day activities of the university. $\operatorname{In}^{19}$ a web-based announcement system is developed. The aim was to provide timely information to students. The development of the system using Java language that can deliver a message from web-based interface (electronic form) and send it to a group of students. The system was closely related with Mobile Notice Board project for the delivery of urgent information to students but could not ascertain a feedback module in the deployment. The author's in ${ }^{6}$ presented a mobile pigeonhole alert system. The authors were motivated to develop a system which allowed for easy and enhanced communication between the administrator-in-charge of messages (office clerk) and the recipients (academic staff) to ensure proper dissemination of information. The aim was to ensure quick notification, delivery and responses to mail in an academic institution. The implementation was accomplished using Java and XML components of Android Software Development Kit (SDK). The system bridged the gap between the administrator and the user of the pigeonhole by ensuring a feedback mechanism to alert the user of a mail when not attended to. However, the system involves human intervention in which the administrator in charge of the mail will have to send notifications to staff. Also training is required when there is a change of administrator on how to use the system

\section{Methodology}

The architecture of the proposed system is presented in Figure 1. The components of the proposed system architecture are presented as follows:

I. Mail Admin: This is the administrator or clerk in charge of dropping the mail in the pigeonhole.

II. Mail Arrival: this is the process in which the mail is dropped in the pigeonhole by the mail admin or a new mail arrived.

III. Light Dependent Resistor and Light Emitting Resistor (LDR/ LER): It is a resistor which has a resistance that varies depending of the light intensity and it is used to turn ON or OFF a device according to the ambient light.

IV. GSM module: An SMS alert is sent to the intended user about the presence of a mail in the pigeonhole.

V. Send Message: The mail messages are sent to the intended mail recipient as coded in the Sensor.

VI. Mail Notification: the user gets a notification message from the GSM module of the presence of a mail in the pigeonhole.

VII. Mail Recipient: This is a user of the pigeonhole who gets a notification message on their mobile phone registered on the sensor informing them of the presence of a mail not yet attended to.

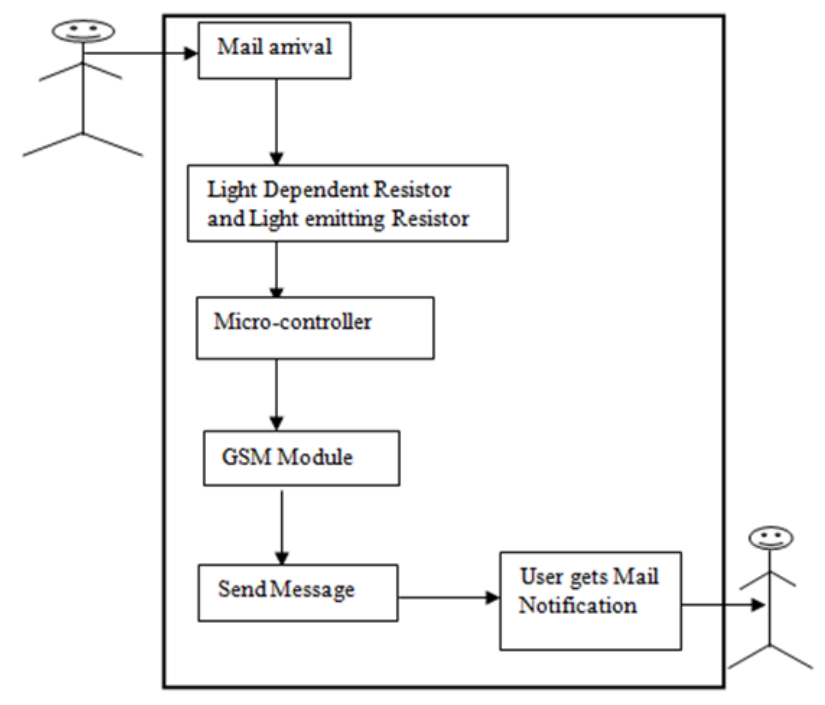

Figure I The architecture of the proposed system.

\section{Mathematical model of the proposed system}

The mathematical model for the proposed system is adopted from ${ }^{6}$ and is presented as follows:

The general idea of the pigeonhole principle states when there are $\mathrm{k}$ pigeonholes and there are $\mathrm{k}+1$ mails, then there will be 1 pigeonhole with at least 2 mails. ${ }^{20}$ The idea sounds trivial but its uses are numerous. Thus, this idea is explored to coin a model that support the implementation of this research work. A conventional 
pigeonhole system consists of one or more pigeonholes for each staff in an institution. Let $p g h$ represents pigeonholes and stff a variable denoting staff.

$$
\forall \text { pgh, } \text { stff }=\{1,2,3 \ldots \mathrm{k}\}
$$

where equation (i) represent one or more pigeonholes for one or more staff while $\mathrm{k}$ is the count number for upper bound value

$$
\forall \text { Alt, } \mathrm{msg}=\{0,1,2,3 \ldots \mathrm{n}\}
$$

where Alt and msg represent alert and message respectively. Equation (ii) indicates zero or more alerts for every message available to the staff in his/her pigeonhole. The map function for the pigeonhole process is presented as follows:

$$
\forall \text { stff : } \exists \text { ! pgh }
$$

indicating that for each staff, there exist exactly one pigeonhole for message delivery

$$
\forall \text { stff : } \exists \mathrm{msg}
$$

equation (iv) denotes that any staff can have one or many messages.

$$
\forall \mathrm{msg}: \exists \text { Alt }
$$

equation (v) denotes that there exist one or many alerts for each message received in the staff pigeonhole.

$$
A l t=\left\{\begin{array}{c}
0, \nexists m s g \\
1, n \exists m s g
\end{array}\right.
$$

$$
R=\left\{\begin{array}{l}
1, \nexists m s g: \forall p g h \\
0, \exists m s g: \forall p g h
\end{array}\right.
$$

where $R$ indicates the staff response to the message available in their pigeonhole.

\section{Circuit Diagram}

The circuit diagram of the proposed system is presented in Figure 2 .This Mobile pigeonhole circuit sends a message to the user about the arrival of a mail. The circuit is powered by a rechargeable battery of 6volt. Once the system is powered, a high bright white light comes up from the Light Emitting Resistor (LER) which is use to trigger the Light Dependent Resistor (LDR) to detect the arrival of a mail in the box. The sensor then sends a signal to the micro-controller which is ATmega8. The micro-controller then sends a signal to the GSM-Module on the arrival of a mail. The GSM-module uses SIM 800 module that is designed as a Data Communication Equipment. It comprises of the ground (GND), the receive data ( $\mathrm{Rx}$ ) which is an input device, the transmit data (Tx) which is an output device and an antenna to detect network signal. Once the micro-controller sends a signal to the GSM-module, the Tx sends a message to the user whose number is registered on the SIM module for a mail arrival in the pigeonhole. The capacitors are used to regulate the frequency of the circuit. When the light from the LER fails to comes up the LDR will become non-conducting and the presence of a mail will not be detected.

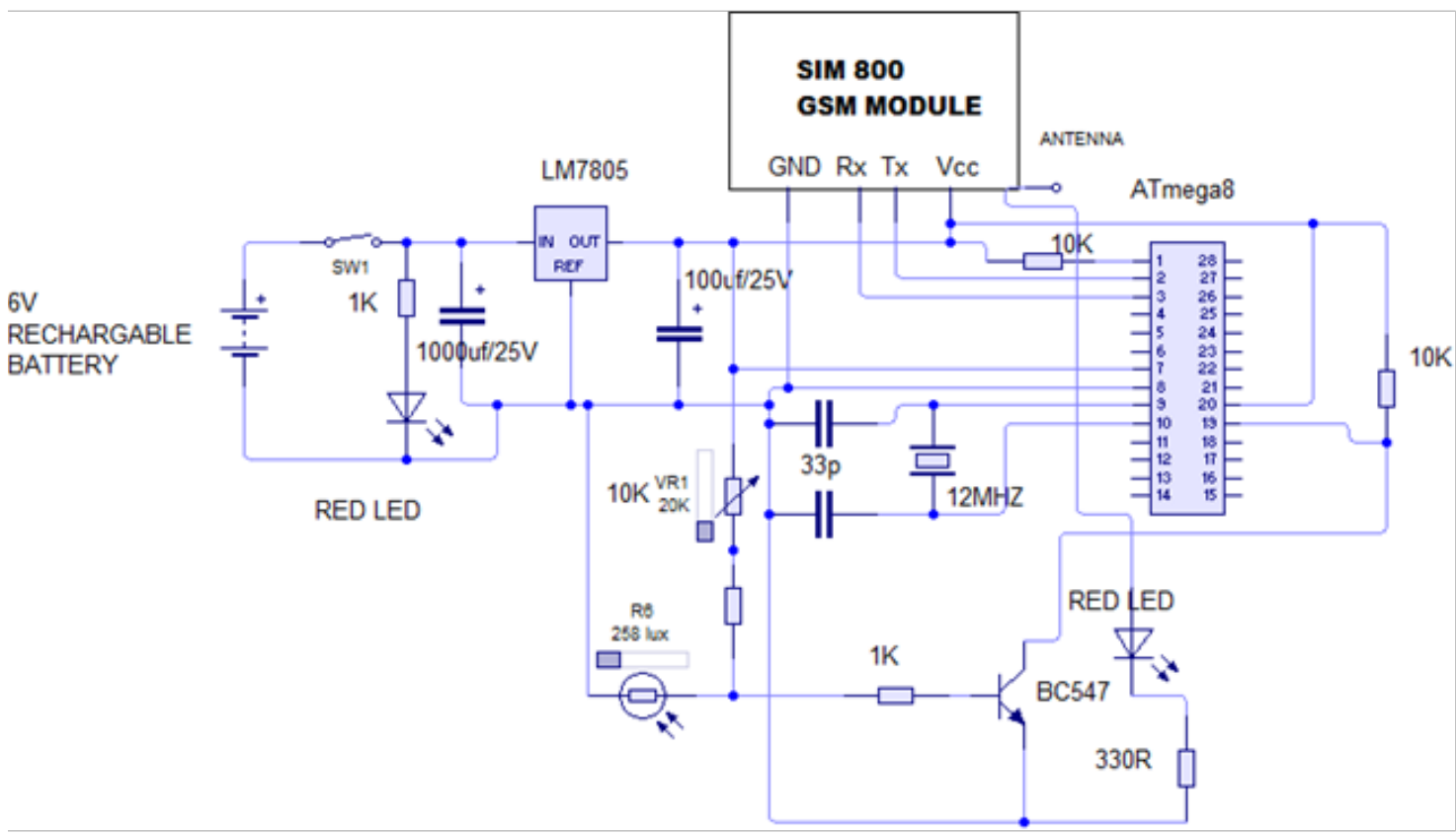

Figure 2 Circuit diagram of the proposed system.

\section{System flowchart}

The system flowchart for the proposed system is presented in Figure 3 .

\section{Implementation}

A. The system development requirements are into two main parts, software and hardware requirements.

B. Software Requirements 

C. JAVA Programming Language
D. Windows 10
E. Ms-Excel

F. Statistical Package for Social Science (SPSS) Software

G. Hardware Requirements

H. HP 620, Pentium (R) Dual-core CPU, 2.90 RAM, 32-bits OS

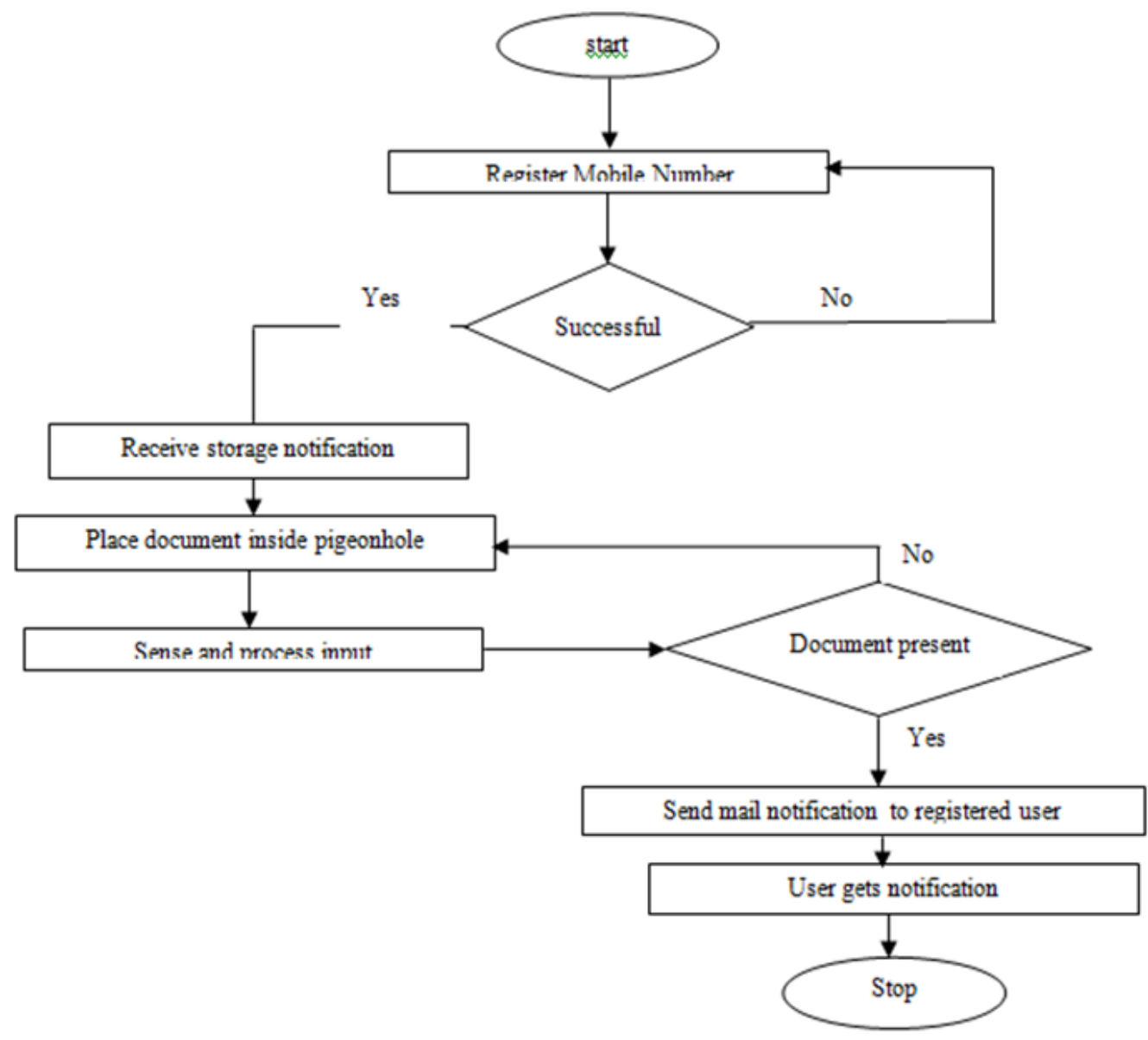

Figure 3 System flowchart.

\section{Mobile number registration}

The mobile pigeonhole system was implemented using a sensor module which consist of mobile number registration, followed by the mobile number storage and acknowledgment and is coded using JAVA Programming Language. For registration of mobile number, the mobile number of the intended user of the pigeonhole is used to send a message "letter" to the SIM number in the GSM module. The user ensures the message is delivered to the receiving number. The word "letter" is a keyword encoded in the sensor module to act as a keyword to store and erase a number in the system memory. This is shown in Figure 4 as follows:

\section{Mobile number storage and acknowledgment}

The mobile number of the user delivered to the sensor module is stored and an ackowledgement message "the number has been stored" is received by the user to show that the number has been received and stored in the GSM moduleas shown in Figure 5 as follows:

\section{Sensor module}

The sensor module consists of several hardware components which works together to ensure the smooth running of the system. Figure 6 shows the sensor module as follows. The components housed on the sensor module include Switch, Battery, Light Emitting Diode, Light Dependent Diode (LDD), GSM Module, Micro-controller, Capacitor, and Transistor.

\section{Pigeonhole interface}

The actual pigeonhole implementation is carried out on both wooden and metal platform for testing.

a) Wooden pigeonhole implementation: Figure 7 shows the implementation of the sensor module using a wooden pigeonhole.

b) Metal pigeonhole implementation: Figure 8 shows the implementation of the sensor module using metal pigeonhole. 


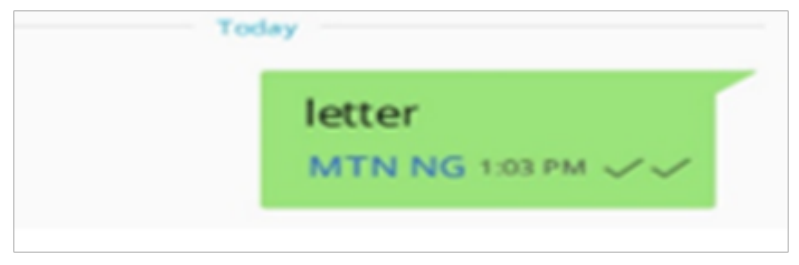

Figure 4 Mobile Interface for number registration.



Figure 5 Mobile Interface for phone number storage.

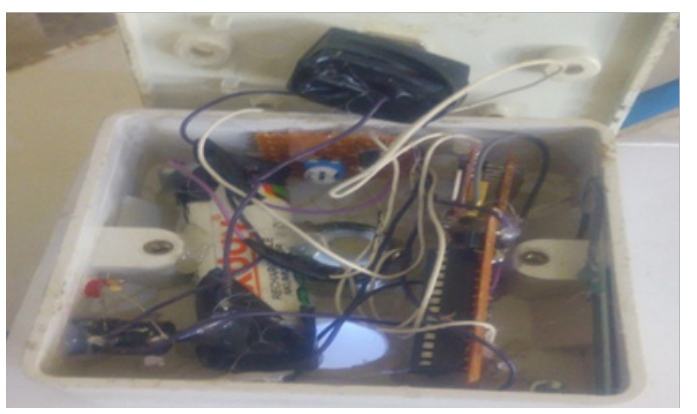

Figure 6 Sensor Module.

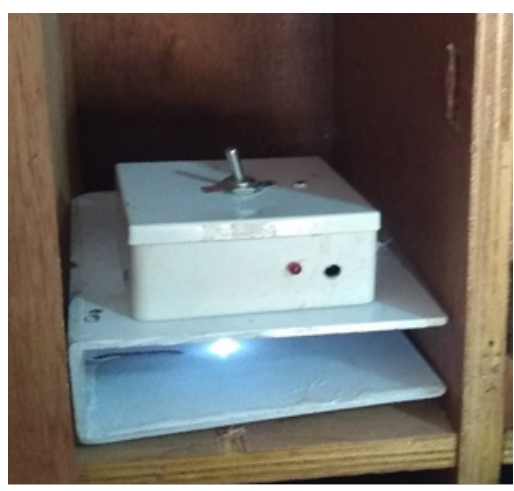

Figure 7 wooden pigeonhole implementation.



Figure 8 Metal pigeonhole implementation.

\section{Data collection}

The system was test on both pigeonhole platforms and a detailed and structured questionnaire was developed and administered to two hundred (200) lecturers of Adekunle Ajasin University in the faculty of Science across nine (9) departments to elicit information about the system performance during testing. The data collected are presented in Table 1 as follows:

$$
\text { Average success rate (ASR) }=\frac{1}{n} \sum_{k=1}^{n} S_{d}
$$

where $n=$ number of lecturers, $S_{d}=$ total successful delivery

$$
(\text { ASR })=92.5 \%
$$

$$
\text { Average failure rate }(\mathrm{AFR})=\frac{1}{n} \sum_{j=1}^{m} F_{d}
$$

where $m=$ number of lecturers and $F_{d}=$ total failed delivery

$$
(\mathrm{AFR})=7.5 \%
$$

\section{Discussion of results}

The data were collected on the system performance which were analyzed using SPSS with the following definitions:

a) Correlation measure (R): show how variables work in relation to each other.

b) Co-variance ( $\mathrm{R}$ square): is the proportion of variability in system testing by the department in the model.

c) Adjusted R square: is a more reliable statistics because it takes in consideration of the sample size

d) Standard error deviation: measures the variability of the actual value from the predicted value.

e) ANOVA: (Analysis of Variance statistics)

f) DF: Degree of freedom

g) F: is to determine if the regression analysis is significant

h) Significant value (Sig): probability that the result occurred randomly

Table 2 shows the descriptive statistics of the given data. It shows the relationship between the central tendency (mean) and the variability (standard deviation). The result shows that over half of the selected respondents buy the idea of the designed system. Table 3 shows the correlation between the departments. The Pearson correlation coefficient shows a positive correlation between the departments and the system because there was no negative value in the result. Table 4 shows the regression coefficients that describes the significant relationship between the independent variable and the dependent variable. In this case, the independent variable is the c computed value for the performance of the system. The computed value denotes the view of the respondent (lecturers) about the systems performance. The independent variable connotes the lecturers who answered the questions that are given during system testing. Therefore, to determine if the system testing is significant, the significant value or the p-value which is 0.013 is used. Since the p-value is lesser than 0.05 , then there is a significant association between the lecturers and their view about the system performance. That is, the objectives of the 
system were achieved. But if the value is greater than 0.05 it means the system did not meet the expected objectives. Table 5 shows the model summary. It is shown that the R Square is $89 \%$, which means that more than almost all the lecturers from each of the departments are in support of the designed system. Table 6 shows the significant of the model. Since the significant value on the table is 0.026 , it shows that the system model is significant since the value is lesser than 0.05 . Table 7 shows the comparative analysis of this work with other existing works. The results derived showed that our system is efficiently analyzed and implemented. Hence, it is an evidence for improvement on other existing results in the reported literatures in Table 7.

Table I data collection

\begin{tabular}{|c|c|c|c|c|c|}
\hline Departments & No of SMS sent & Successful delivery & Failed delivery & $\%$ of Success rate & $\%$ of Failure rate \\
\hline Computer science & 24 & 23 & 1 & 95.8 & 4.2 \\
\hline Physics and electronics & 21 & 18 & 3 & 85.7 & 14.3 \\
\hline Plant Science \& Biotech & 27 & 25 & 2 & 92.5 & 7.5 \\
\hline Microbiology & 17 & 17 & 0 & 100 & 0 \\
\hline Biochemistry & 25 & 23 & 2 & 92 & 8 \\
\hline Mathematics/Ind. Math & 20 & 17 & 3 & 85 & 15 \\
\hline Animal \& Env. Biology & 22 & 21 & I & 95.5 & 4.5 \\
\hline Chemical Science & 26 & 24 & 2 & 92.3 & 7.7 \\
\hline Earth Sciences & 18 & 17 & I & 94.4 & 5.6 \\
\hline TOTAL & 200 & 185 & 15 & 833.2 & 66.8 \\
\hline
\end{tabular}

Table 2 Descriptive statistics

\begin{tabular}{lll}
\hline Mean & Std. Deviation & $\mathbf{N}$ \\
\hline 2.3827 & 0.29829 & 200 \\
5 & 2.55841 & 200 \\
\hline
\end{tabular}

Table 3 Correlation Measure

\begin{tabular}{llll}
\hline & & System testing & Department \\
\hline Pearson & system_testing & $\mathrm{I}$ & 0.774 \\
Correlation & Department & 0.774 & $\mathrm{I}$ \\
& system testing & $\cdot$ & 0 \\
Sig. (I-tailed) & Department & 0 & $\cdot$ \\
& system_testing & 200 & 200 \\
N & Department & 200 & 200 \\
\hline
\end{tabular}

Table 4 Regression Coefficients

\begin{tabular}{llllll}
\hline \multirow{2}{*}{ Model } & \multicolumn{2}{l}{ Unstandardized coefficients } & $\begin{array}{l}\text { Standardized } \\
\text { coefficients }\end{array}$ & t & Sig. \\
\cline { 2 - 5 } & B & Std. error & Beta & 30.633 & 0.013 \\
\hline (Constant) & 1.931 & 0.063 & & 8.024 & 0 \\
\hline
\end{tabular}

Dependent Variable: system_testing

Table 5 Model Summary

\begin{tabular}{lllll}
\hline Model & $\mathbf{R}$ & R Square & Adjusted R square & Std. error of the estimate \\
\hline & $.774 a$ & 0.89 & 0.59 & 0.19094 \\
\hline
\end{tabular}

b) Predictors: (Constant), department 
Table 6 ANOVA

\begin{tabular}{lllllll}
\hline Model & & R & Df & Mean square & F & Sig. \\
\hline & Regression & 2.347 & I & 2.347 & 64.38 & $.026 \mathrm{~b}$ \\
I & Residual & 1.568 & 43 & 0.036 & & \\
& Total & 3.915 & 44 & & & \\
\hline
\end{tabular}

Dependent Variable: system_testing

Predictors: (Constant), department

Table 7 Comparison of current work with the result of the existing works

\begin{tabular}{|c|c|c|c|c|c|}
\hline Author & Sensor & Messaging mode & Platform & Programming language & Pigeonhole \\
\hline Mohammad \& Norhayati ${ }^{7}$ & Not used & SMS & Software & VB & None \\
\hline Markett et al., ${ }^{8}$ & Not used & SMS & Software & JAVA & None \\
\hline Bin Hajj Sidek., ${ }^{5}$ & Not & SMS & Software & Rational Unified Process & None \\
\hline Manguni et al., ${ }^{12}$ & Not used & SMS & Software & JAVA & None \\
\hline Krishna et al. ${ }^{14}$ & Not used & SMS & Software & DBMS & None \\
\hline Abdullah., ${ }^{15}$ & $\begin{array}{l}\text { Infrared/ } \\
\text { Ultrasonic }\end{array}$ & SMS & Hardware & $\mathrm{C}++$ & Metal \\
\hline Wahab et al., ${ }^{16}$ & IR /LED & SMS & Software & None & Metal \\
\hline Ajayi et al., ${ }^{6}$ & Not used & SMS & Software & JAVA & Wooden \\
\hline Current research & LED/LDD & SMS & $\begin{array}{l}\text { Hardware/ } \\
\text { Software }\end{array}$ & JAVA & Wooden/ Metal \\
\hline
\end{tabular}

\section{Conclusion}

Information system is an essential aspect of any organization which help with the dissemination and retrieval of information among members. An organization increasingly adopt information technology to move their operations and services to a new level by embracing information systems to ease information dissemination within the organization. Most institutional organizations achieve this through the use of pigeonhole as a message box to manage information available every staff member of the organization. Hence, the designed system has been able to efficiently ease the work of office clerks as well as the pigeonhole users by reducing the stress of daily pigeonhole checkin. It provided a platform that allows quick feedback mechanism for smooth record and operation to urgent mails.

\section{Acknowledgments}

None.

\section{Conflicts of interest}

Author declares that there is no conflict of interest.

\section{Rreferences}

1. Shaqiri BA.Management Information System and Competitive Advantage. Mediterranean Journal of Social Sciences. 2015;6(1):204208.

2. Adeoti-Adekeye WB. The Importance of Management Information Systems. Library Review, MCB University Press; 1997;46(5):318-
327

3. Al-Mamary YH, Shamsuddin A, Aziati N. The Meaning of Management Information Systems and its Role in Telecommunication Companies in Yemen. American Journal of Software Engineering. 2014;2(2):22-25.

4. Bin Haji Sidek SF. The development of the Short Messaging Service (SMS) application for the school usage. Proceedings 2010 International Symposium on Information Technology - System Development and Application and Knowledge Society, ITSim'10, 3. 2010. p. 1382 1386.

5. Ensour HS, Alinizi TM. The Impact of Management Information Systems (MIS) Technologies on the quality of Services provided at the University Of Tabuk. International Journal of Network Security \& Its Applications (IJNSA). 2014;6(2):1-20.

6. Ajayi OO, Adetuyi TE, Omomule TG, et al. Design and Implementation of Mobile Pigeon-Hole Alert System (PHAS). Communications on Applied Electronics (CAE). 2018;7(16):10-16.

7. Mohammad MA, Norhayati A. A Short Message Service for Campus Wide Information Delivery. Proceeding of 4th National Conference on Telecommunication Technology. 2003. p. 216-221.

8. Markett C, Sanchez IA, Weber S, et al. Using Short Message Service to encourage Interactivity in the Classroom. Computers \& Education. 2005; 46(2006). 280-293

9. Shahjahan MD, Kafi M, Nahin M, et al. An Implementation of OnLine Traffic Information System via Short Message Service (SMS) for Bangladesh. IEEE International Joint Conference on Neural Networks (IEEE World Congress on Computational Intelligence. 2008:26122618 . 
10. Jamil S, Mousumi FA. Short Messaging Service (SMS) Based m-Banking System in Context of Bangladesh. Proceedings of 11th International Conference on Computer and Information Technology (ICCIT 2008). 2008. p. 599-604.

11. Katankar VK, Thakare VM. Short Message Service using SMS Gateway International Journal on Computer Science and Engineering (IJCSE). 2010;2(4):1487-1491.

12. Manguni Jr, Navarro R, Rosario ML, et al. ChitSMS: Community Health Information Tracking System Using Short Message Service. 3rd International Conference on Human-Centric Computing. 2010:1-6.

13. Okuboyejo SR, Ikhu-Omoregbe NA, Mbarika VW. A Framework for the Design of a Mobile-Based Alert System for Outpatient Adherence in Nigeria. African Journal of Computing \& ICT. 2012;5(5):151-158.

14. Krishna V, Anurag R, Prabhune SS. Short Messaging Service as an Alternative for Pushing Information to build efficient Information Passing Systems in Academic Institutions. Proceedings of the 2014 Conference on IT in Business, Industry and Government: An International Conference by CSI on Big Data, CSIBIG; 2014. p. 1-6.

15. Abdullah NB. Smart pigeonhole system by sending notification through short messaging system". A thesis submitted to the Faculty of Computer Systems and Software Engineering. 2015.
16. Wahab MHA, Al' Hafiz Riman A, Kadir HA, et al. GSM-Based Notification System for Electronic Pigeon Hole. International Conference on Networked Digital Technologies (NDT) 2010: Networked Digital Technologies. 2016:619-630.

17. Obea JV, Pardo I, Villa I, et al. SMS, A New Tool in the Radiological Information System Information Exchange. 2004

18. Wahab MHA, Nor DM, Mutalib AA, et al. Development of Integrated e-parcel Management System through GSM Network. 2nd International Conference on Interaction Science 2009, November 24-26. Seoul, Korea. 2009:1-6.

19. Curran K, Craig R. A Short Message Service Online Application for Delivering Urgent Information to Students. 1st Joint IEI/IEE Symposium on Telecommunications Systems Research. 27th November 2001, IEI Headquarters, Clyde Rd, Dublin. 2001.

20. Sengothai R. Generalized Pigeon Hole Principle and its Applications. International Journal on Recent and Innovation Trends in Computing and Communication (IJRITCC). 2016;4(11):19-21. 